\title{
The Origin and Interpretation of Line Profile Variations of Pulsating B Stars
}

\author{
J. De Ridder, C. Aerts ${ }^{1}$ \\ Instituut voor Sterrenkunde, Katholieke Universiteit Leuven, \\ Celestijnenlaan 200 B, B-3001 Heverlee, Belgium
}

\begin{abstract}
We report on an ongoing study on the importance of the contributions of different photospheric variations to the line profile variations of hot pulsating stars.
\end{abstract}

\section{Introduction}

Almost 30 years after Osaki (1971) proposed a successful nonradial pulsation code that mimicked the basic properties of the varying line shape of the $\beta$ Cephei stars, we still do not have a satisfactory understanding of the impact of, e.g., temperature variations. Meanwhile, the Slowly Pulsating B stars (SPBs) joined the group of pulsating B-type stars, also showing line profile variations, but this time originating from a photosphere dominated by horizontal motions. No detailed effort to model the observed variations of such stars has been done so far. To model line profile variations of observed pulsating B stars, we calculate line profiles which are subject to 5 different time-dependent variations: the velocity field, the surface brightness variation, the equivalent width variation, the thermal broadening variation and the geometrical distortion of the surface. We take both radial and horizontal motions into account. We also look at, and make an inventory of, the observed $\mathrm{EW}$ variations and $T_{\text {eff }}$ variations.

\section{The Model}

We divide the stellar surface in small cells. For each of the cells, we compute the time-dependent local line profile. We then integrate over the visible stellar surface to obtain the observed line profile. As a first approximation we used Gaussian local line profiles which are shifted because of the velocity of the cell due to pulsation and rotation. The Gaussians have a certain width, equivalent width and a continuum which are all treated as time dependent. We make the following approximations:

Velocity field: We assume one nonradial spheroidal mode, not modified by rotation and slow uniform rotation.

Geometrical distortions: We take the time-dependence of both the area and the

\footnotetext{
${ }^{1}$ Postdoctoral Fellow, Fund for Scientific Research, Flanders
} 
orientation of each visible cell into account using both radial and horizontal displacement.

Intensity variation: Looking at the temperature dependence of the continuum at the relevant wavelengths, given by Kurucz models, we adopt $\delta I_{\lambda} / I_{\lambda}=1.9 \delta T / T$ $\sim Y_{l}^{m} e^{i 2 \pi f t}$.

Local width variation: From classical broadening theory we learn that thermal Doppler broadening results in a Gaussian profile with a width $\sigma \sim \sqrt{T}$, so that $\delta \sigma / \sigma=1 / 2 \delta T / T$.

Local $E W$ variations: Because the temperature changes, the local $\mathrm{EW}$ will also change due to ionisation and recombination. We use the theoretical NLTE EW data of Becker \& Butler (1990) to model the EW of the Si III $(455.3 \mathrm{~nm})$ line.

The amplitudes of the relative radius variation and of the temperature variation are taken as free parameters, but care has been taken never to violate the observed amplitudes of the radial velocity and the equivalent width variations. In the near future we plan to use the eigenfunctions resulting from a nonadiabatic pulsation code, instead of free parameters.

\section{Observational Constraints}

We looked at the $T_{\text {eff }}$ variation of 12 SPBs and $15 \beta$ Cephei stars. We used

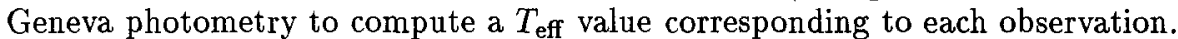
Throughout, the amplitude was estimated as $\sqrt{2} \sigma$, where $\sigma$ is the standard deviation of the $T_{\text {eff }}$ time series. We only used stars for which at least 50 measurements were available. We find that the relative amplitude of the $T_{\text {eff }}$ variation lies between $0.5 \%$ and $2 \%$ for the studied SPBs and between $1 \%$ and $3 \%$ for the studied $\beta$ Cephei stars.

We also looked at the EW variation of $5 \beta$ Cephei stars (Si III $455 \mathrm{~nm}$ ) and 17 SPBs (Si II $413 \mathrm{~nm}$ ). We estimated the amplitude in the same way as for the $T_{\text {eff }}$ variation. We observe amplitudes of the relative $\mathrm{EW}$ variations to be between $2 \%$ and $12 \%$.

\section{Results}

The main results for the $\beta$ Cephei stars are the following. For realistic values of the parameters, the velocity field remains the dominant source of line profile variations. Often the modifications by the extra variations would currently not be detectable in practice. Of all the extra variations included, the local EW variation seems to be the most important one. Next is, to a lesser extent, the intensity variation. The width variation and the geometrical distortions seem to be negligible. For high degree, equator-on sectoral modes, the bumps can appear more pronounced due to temperature effects.

\section{References}

Becker, S. R. \& Butler, K. 1990, A\&AS, 84, 95

Osaki, Y. 1971, PASJ, 23, 485 